A N N A L E S Annales de Bretagne et des Pays de l'Ouest

\title{
Martin, Philippe et Henryot, Fabienne (dir.), Dom Augustin Calmet. Un itinéraire intellectuel.
}

\section{Marie Berthelot}

\section{(2) OpenEdition}

1 Journals

Édition électronique

URL : http://journals.openedition.org/abpo/1959

DOI : $10.4000 /$ abpo.1959

ISSN : 2108-6443

Éditeur

Presses universitaires de Rennes

\section{Édition imprimée}

Date de publication : 10 avril 2011

Pagination : 201-203

ISBN : 978-2-75351410-2

ISSN : 0399-0826

\section{Référence électronique}

Marie Berthelot, « Martin, Philippe et Henryot, Fabienne (dir.), Dom Augustin Calmet. Un itinéraire intellectuel. », Annales de Bretagne et des Pays de l'Ouest [En ligne], 118-1 | 2011, mis en ligne le 09 mai 2011, consulté le 22 septembre 2020. URL : http://journals.openedition.org/abpo/1959 ; DOI : https:// doi.org/10.4000/abpo.1959 
empire mondial, et l'oligarchie lombarde, qui veut tirer profit de sa participation à l'administration d'une partie de cet ensemble. Les expérimentations du milieu du $\mathrm{XVII}^{\mathrm{e}}$ siècle, relativement novatrices comparées à ce qui se passe alors en France par exemple, sont entravées par un contexte militaire et fiscal très défavorable. Elles ne donneront leurs fruits qu'une fois la paix revenue, dans le dernier tiers du xvII siècle et le début du xvIII.

Stéphane PERRÉON

Martin, Philippe et Henryot, Fabienne (dir.), Dom Augustin Calmet. Un itinéraire intellectuel, Paris, éditions Riveneuve, 2008, 428 p.

Ce livre rassemble les Actes du colloque organisé les 18-20 novembre 2007 par le Centre de Recherche Universitaire Lorrain en Histoire, en association avec les villes de Nancy et Senones, à l'occasion du $250^{\circ}$ anniversaire de la mort de dom Augustin Calmet (1672-1757), religieux bénédictin de la Congrégation de Saint- Vanne implantée en Lorraine. Celle-ci accorde une grande place aux études et à l'érudition (théologie, exégèse, histoire...), tout comme la Congrégation de Saint-Maur fondée simultanément en France sur son modèle. II n'y a pas lieu de rappeler la part prise par les mauristes dans I'historiographie bretonne aux lecteurs des ABPO. Ceux-ci ne manqueront pas d'établir des parallèles significatifs avec la situation en Lorraine où se retrouvent les enjeux analogues propres à l'écriture d'une histoire provinciale. L'histoire des provinces, alors en développement, est bien souvent au service d'une cause politique. II faut composer entre les exigences des institutions, celles de la noblesse locale et la nécessité de ne pas déplaire à la couronne. Les religieux ont bien conscience de ces enjeux. Leur désir d'impartialité rencontre des obstacles. Des rapports de forces s'établissent entre les puissants et les érudits. On sait que dom Lobineau, chargé par les États de Bretagne de rédiger une Histoire de la Bretagne ne put réaliser le troisième volume initialement prévu du fait de l'intervention des Rohan. Ces derniers demandent à dom Morice de réviser l'oeuvre de son prédécesseur et d'achever son travail selon leurs volontés ${ }^{1}$. A la même époque, dom Calmet se voit confier en 1719, par le duc Léopold la réalisation d'une histoire de la Lorraine, après l'abandon du projet par un autre religieux. II n'a cependant pas le titre officiel d'historiographe. Son Histoire civile et ecclésiastique de la Lorraine (1728-1729), dont certains passages ont l'heur de déplaire au pouvoir politique doit elle aussi subir des remaniements.

Ces Actes sont matière à d'autres rapprochements significatifs avec les milieux de l'érudition monastique au temps de la " sécularisation des savoirs ». En effet, " ce colloque a été l'occasion de constater un paradoxe : I'indéniable célébrité de l'érudit bénédictin va de pair avec de nombreux préjugés à son endroit, et plus encore de nombreuses zones d'ombres entourant le personnage » [p. 7]. II ne s'agit pas de proposer une biographie classique mais d'étudier le personnage de dom Calmet à partir de cinq angles d'approche: "Dom Calmet en ses miroirs », "Dom Calmet et l'ordre de saint Benoît », "Dom Calmet et les sciences sacrées », " Dom Calmet et I'histoire lorraine», «Dom Calmet et ses lecteurs ».

1. QUÉNIART, Jean, « Les mauristes et l'historiographie bretonne » dans Chroniqueurs et historiens de la Bretagne du Moyen Âge au milieu du xx siècle, Rennes, PUR, coll. « Histoire », 2001, p. 111-123. Dom Lobineau publie son Histoire de Bretagne en 1707. L'ouvrage de dom Morice est publié en 1750, le second tome, réalisé par dom Taillandier, en 1756. 
L'ordre bénédictin est alors florissant. Dom Calmet vit lors d'une période traversée par des débats (crise janséniste, émergence des idées des Lumières) et marquée par des évolutions en matière d'érudition (mutation de la critique biblique, renouvellement des méthodes historiques). Dom Calmet, exégète et historien ancré dans son temps, s'intéresse à toutes ces questions. Son œuvre est abondante : 56 ouvrages dans divers domaines. Sa vie se divise en quatre étapes : sa formation (jusqu'en 1704), son séjour à Paris (1706-1716), son ancrage en Lorraine (1718-1729), son abbatiat à Senones (1729-1757). Il possède une vocation d'érudit et nullement celle de la charge d'âmes ou de la prédication : les caractéristiques de la Congrégation de Saint-Vanne répondent à ses aspirations, il est profondément attaché à cette dernière. II y exerce diverses responsabilités dont celle d'abbé. Son séjour à Paris (au couvent des Blancs-Manteaux) est très important dans son itinéraire. II y rencontre les personnalités influentes de l'époque comme dom Mabillon. II commence à tisser des liens avec les libraires. Dom Calmet est ainsi intégré à la "République des Lettres »; au fil du temps, il se construit un étroit réseau de correspondants et de collaborateurs.

En matière d'exégèse, il souhaite fournir des clefs de lecture à ceux qui s'intéressent à l'Écriture Sainte. S'il est acquis un temps aux idées jansénistes, il fait preuve de prudence dans ses écrits et adopte toujours des positions orthodoxes. II fait preuve d'exigence et de volonté critique. II interprète le texte biblique de façon littérale. II se veut non pas novateur, mais harmonisateur. Si, parfois, son œuvre est novatrice, la plupart du temps elle est empreinte de conceptions traditionnelles. II est " fidèle à une conception selon laquelle il existait un bloc orthodoxe autour duquel tout pouvait être discuté, marges où il pouvait faire des concessions ou faire preuve d'originalité [...]. Cependant nul ne pouvait remettre en cause le bloc fondamental » [p. 159]. Ses travaux répondent à une volonté de vulgarisation, il rédige ses ouvrages en français. Son œuvre n'est pas seulement synthétique, il prend position dans les débats de son temps. Le succès auprès du public est important, il est sollicité par les libraires. Toutefois, il s'attire les critiques aussi bien des philosophes des Lumières (Voltaire, notamment) que celles d'autres religieux.

L'histoire passionne alors le public. Un grand nombre de ses contemporains perçoivent dom Calmet comme un grand historien. II publie trente et un livres en ce domaine, tant des ouvrages d'histoire religieuse que d'histoire profane. Figure emblématique de l'érudition bénédictine dans la lignée de dom Mabillon, il conçoit I'histoire à diverses échelles, du local à l'universel, et souhaite se démarquer de la tradition des annales et des chroniques. II se rend dans les bibliothèques et les lieux d'archives pour ses recherches et se fait aider de collaborateurs. II fait montre de sens critique et de rigueur. Mais, parfois, ne vérifiant pas ses sources, il commet des erreurs, et se montre partial. Ses travaux sur l'histoire lorraine sont reconnus aujourd'hui encore, même s'il convient de les utiliser avec précaution.

Les lecteurs des ouvrages de dom Calmet se divisent en trois groupes : les bénédictins, les autres religieux et les laïcs au courant des publications récentes. Ses lecteurs sont avant tout des bénédictins, dont de nombreux membres de sa congrégation; très peu de religieux d'autres ordres ou de séculiers. Ses ouvrages sont peu présents dans les bibliothèques des ordres mendiants en Lorraine. II rencontre le succès auprès des élites bourgeoises lorraines. Grâce à ses liens avec de nombreux libraires, la diffusion de ses ouvrages est large, en particulier vers le monde germanique et l'Europe centrale. Sa volonté de vulgarisation, à laquelle l'écriture en français participe lui permet de toucher un public varié, y compris des femmes. Le public se prête à divers types de lectures : érudite, critique, polémique. Son œuvre éclectique est diversement appréciée par ses contemporains. S'ils sont nombreux à reconnaître son ampleur, ils mentionnent aussi ses failles (tendance à la 
compilation, précipitation, naïvetés...). Ses traités d'exégèse connaissent un grand succès tout au long du XvIII siècle; par la suite, son influence décroît, en lien avec les renouvellements de la discipline.

Par les divers angles d'approche abordés, les vingt-deux contributions réunies dans ces Actes répondent aux objectifs affichés de ce colloque en proposant une vision renouvelée du sujet. Dom Calmet « est la manifestation d'un monde clérical qui releva, parfois avec maladresse, toujours avec fougue, les défis d'une époque. Il est à la fois l'héritier de siècles d'érudition et de lente maturation du savoir, et le clerc inséré dans les réseaux de son époque. Ni crédule ni moderne, il est la figure emblématique d'un monachisme qui tenta de s'adapter au monde de "la crise de conscience européenne" » (p. 11). L'ouvrage est dépourvu de conclusion, mais la préface, très claire, permet d'emblée d'avoir une vision synthétique de la question et chaque partie, à l'exception de la première, s'ouvre sur sa propre introduction. Une chronologie récapitule les grandes étapes de la vie de dom Calmet en y associant ses principales publications. Toujours replacées dans leur contexte historique, ces informations permettent de restituer l'originalité de la pensée de dom Calmet, mais aussi de rapporter ses positions à celles de ses pairs et de ses contemporains.

Marie BERTHELOT étudiante en Master 2 HSC - Université de Rennes 2 Haute-Bretagne

Mоuнot, Jean-François, Les réfugiés acadiens en France. 1758-1785 L'impossible réintégration?, Québec, Septentrion, 2009, 448 p.

Cet ouvrage est issu d'une thèse de doctorat réalisée en 2006, à l'Institut universitaire européen de Florence. Jean-François Mouhot y décrit I'accueil des Acadiens dans les ports français, à la suite du "Grand Dérangement » de 1755. II explique la situation particulière de ces exilés qui subissent deux déportations, d'abord en 1755 lorsqu'ils sont répartis dans les Treize Colonies d'Amérique, puis en 1758, à la suite de la prise de Louisbourg, quand les premiers acadiens arrivent en France. Ce travail vient enrichir l'historiographie des questions acadiennes dans laquelle il faut compter les ouvrages pionniers d'Ernest Martin et de Gérard-Marc Braud sur les Acadiens en Bretagne. Outre les archives officielles du gouvernement royal, Jean-François Mouhot utilise des correspondances privées d'Acadiens, mais aussi celles des administrateurs, ainsi que des archives judiciaires.

L'ouvrage s'ouvre sur une évocation des relations particulièrement conflictuelles existant alors entre Français et Britanniques. Le site acadien, de faible étendue, se place au coeur des liaisons entre Londres et le continent américain (Massachussetts et les bancs de pêche de Terre-Neuve). Le contexte politique modifie une première fois la situation des Acadiens au moment du traité d'Utrecht (1713) puis lors des premiers temps de la Guerre de Sept ans. Le conflit franco-britannique débute en 1756 mais dès juillet 1755, le gouverneur américain, Charles Lawrence, ordonne l'expulsion de tous les habitants d'origine française de sa juridiction. Jean-François Mouhot a choisi de ne pas exposer en détail le périple des Acadiens dans les Treize Colonies mais de s'intéresser aux conditions d'existence des Acadiens à partir de 1758, quand ils sont expulsés de lîle Royale, de l'île Saint-Jean et des prisons de Grande-Bretagne, vers les ports français, de Saint-Malo, Morlaix, La Rochelle et Cherbourg.

Qu'advient-il des Acadiens quand ils arrivent en France? Dans un premier temps, l'auteur évoque les différents projets d'établissement français proposés par l'admi- 